\title{
Empagliflozin improves diabetic renal tubular injury by alleviating mitochondrial fission via AMPK/SP1/PGAM5 pathway.
}

\author{
Xiangyang Liu ${ }^{1}$, Chaofei Xu${ }^{1}$, Linxin $\mathrm{Xu}^{1}$, Xiao-yu Li ${ }^{1}$, Hongxi Sun ${ }^{1}$, Mei Xue ${ }^{1}$, Ting $\operatorname{Li}^{1}$, \\ Xiaochen $\mathrm{Yu}^{1}$, Bei $\mathrm{Sun}^{1}$, and Liming $\mathrm{Chen}^{1}$ \\ ${ }^{1}$ Tianjin Medical University Hospital for Metabolic Diseases
}

May 5, 2020

\begin{abstract}
Background and Purpose: Excessive mitochondrial fission was observed in diabetic kidney disease (DKD). Phosphoglycerate mutase family member 5 (PGAM5) plays an important role in mitochondrial fission by dephosphorylating the DRP1S637. Whether PGAM5 participates in the mitochondrial fission in diabetic renal tubular injury is unknown. Clinical trials have observed encouraging effect of Sodium-glucose cotransporter 2 (SGLT2) inhibitors on DKD though the underling mechanisms remain unclear. Experimental Approach: We use KK-Ay mice as diabetic model and Empa was administrated by oral gavage. The mitochondrial fission and expressions of p-AMPK, SP1, PGAM5 and DRP1-S637 were tested. We also examined these changes in HK2 cells that cultured in normal glucose (NG), high glucose (HG) and high glucose+Empa (HG+Empa) environment. Then we verified our deduction using AMPK activator, inhibitor, si-SP1 and si-PGAM5. Lastly, we testified the interaction between SP1 and the PGAM5promotor by CHIP assay. Key Results: The mitochondrial fission and the expression of SP1, PGAM5 increased and the expression of p-AMPK, DRP1-S637 decreased in diabetic or HG environment. These changes were all reversed in Empa or AICAR treated groups. These reversal effect of Empa could be diminished by Compound C. Either si-SP1 or si-PGAM5 could alleviated the mitochondrial fission without affection on AMPK phosphorylation. Finally, the CHIP assay confirmed the interaction between SP1 and the PGAM5 promotor. Conclusions and Implications: The PGAM5 aggravates the development of diabetic renal tubular injury and the Empa can improve the DKD by alleviating mitochondrial fission via AMPK/SP1/PGAM5 pathway.
\end{abstract}

\section{Introduction}

Diabetic kidney disease (DKD) is the leading cause of end-stage renal disease in the United States (Reidy, Kang, Hostetter \& Susztak, 2014). Although chronic hyperglycemia has been considered as the main culprit for microvascular complications of diabetes, the underlying mechanism of DKD remains poorly understood (Rossello \& Yellon, 2017). Current strategies show limited efficacy in preventing the progression of DKD, such as control of blood pressure and glucose levels along with administration of angiotensin-receptor blockers (ARBs) and angiotensin-converting enzyme inhibitors (ACEI) (Gregg et al., 2014). These findings remind us that there may be some unknown mechanisms that involved in the development of DKD.

Emerging evidence has showed that the proximal tubule plays a pivotal role in the initiation and progression of DKD (Russo, Sandoval, Campos, Molitoris, Comper \& Brown, 2009). The kidney, especially the proximal tubules is high-energy demanded and rich of mitochondria (Weinberg, Venkatachalam, Roeser \& Nissim, 2000). Recent studies have confirmed the involvement of mitochondria fission in the development of DKD (Bhargava \& Schnellmann, 2017). As a result of loss of mitochondrial membrane potential, fission is induced by the translocation of dynamin-related protein 1 (DRP1) from the cytosol to the mitochondrial outer membrane (Bhargava \& Schnellmann, 2017). Bunch of studies have suggested the posttranscriptional 
phosphorylation of Drp1 as a key modulator of mitochondrial fission. Phosphorylation at Serine-616 is associated with increased activity of DRP1 (pro-fission). On the contrary, phosphorylation at Serine-637 shows opposite effect (anti-fission) (Chang \& Blackstone, 2007). However, the underlying molecular mechanism for mitochondrial dynamics regulation in DN are not fully understood.

Recent studies showed that metformin and 5-aminoimidazole-4- carboxamide-1-riboside (AICAR) could attenuate DKD in vitro and in vivo experiments by activating Adenosine 5'-monophosphate activated protein kinase-(AMPK) (Lee et al., 2013; Takiyama et al., 2011). Meanwhile, AMPK was reported as a regulator of mitochondrial homeostasis via inhibiting mitochondrial fission in several studies (Toyama et al., 2016; Wang et al., 2017). So, we hypothesize that the renal protective effect of AMPK was correlated with its anti-fission function, but the underling mechanisms remained unclear.

Phosphoglycerate mutase family member 5 (PGAM5) plays an important role in the initiation of mitochondrial fission by dephosphorylating the DRP1S637 and promoting DRP1 mitochondrial translocation (Wang, Jiang, Chen, Du \& Wang, 2012). PGAM5 is a mitochondria-resident protein. Bunch of studies have confirmed its involvement in the development of Parkinson's disease ( $\mathrm{Lu}$ et al., 2014), acute kidney injury (Gu et al., 2019) and liver inflammation (Kang et al., 2015). However, whether the PGAM5 participates in the development of DKD and if the AMPK involved in the regulation of PGAM5 in DKD are unknown.

Sodium-glucose cotransporter 2 (SGLT2) inhibitors were recently developed as a class of anti-diabetic agents to promote urine glucose excretion without changing the secretion of insulin (Steven et al., 2017; Zinman, Lachin \& Inzucchi, 2016). Clinical trials have observed encouraging effect of SGLT2 inhibitors on improving DKD outcomes (Neal, Perkovic \& Matthews, 2017; Perkovic et al., 2019; Wanner, Inzucchi \& Zinman, 2016), though the underlining mechanisms are remained controversial. Recent evidence showed that the SGLT2 inhibitors, Empagliflozin (Empa), can activate AMPK and alleviate the mitochondrial fission in diabetic myocardial microvascular endothelial cell through recovering the decreased phosphorylation of DRP1 at the Serine-637 (Zhou, Wang, Zhu, Hu, Chen \& Ren, 2018), suggesting the possible involvement of PGAM5. Since the proximal tubules are the main site of SGLT2 expression and rich of mitochondria (Washburn \& Poucher, 2013), we hypothesize that the Empa could fulfill its renal protective effect by alleviating mitochondrial fission via prompting the AMPK phosphorylation in DKD and the PGAM5 may be involved in this procedure.

\section{Materials and Methods}

\subsection{Animal Experimental Design}

All experiments described were approved by the Animal Ethical and Experimental Committee of the Tianjin Medical University and performed in accordance with their guideline. Animal studies are reported in compliance with the ARRIVE guidelines (Kilkenny, Browne, Cuthill, Emerson \& Altman, 2010; McGrath \& Lilley, 2015) and with the recommendations made by the British Journal of Pharmacology. All study animals were purchased from HFK Bioscience Co. Ltd, Beijing. Fifteen 8-week-old female C57BL/6J wild-type mice (average weight $19.4 \pm 3.2 \mathrm{~g}$ ) and 30 spontaneous diabetic KK-Ay mice (average weight $25.6 \pm 3.6 \mathrm{~g}$ ) were used in this study. Lot of studies have confirmed that KK-Ay mice developed proteinuria, mesangial matrix accumulation and GBM thickening, which validated this strain as a good model for studying DKD (Ito et al., 2006). Animals were housed in Specific Pathogen Free facilities with maintained temperature ( $23+-1 \operatorname{degC})$ and humidity under 12/12-h light-dark cycle. Mice were maintained in cages with a maximum of four mice per cage. The C57BL/6J mice were fed with regular chow that comprises of $5 \%$ fat, $53 \%$ carbohydrate and $23 \%$ protein, and the KK-Ay mice were fed with high-fat diet that consists of $17.9 \%$ fat, $48 \%$ carbohydrate and $17.5 \%$ protein. All the mice could drink water freely. Mice were given analgesia immediately preceding surgery with buprenorphine $(0.1 \mathrm{mg} * \mathrm{~kg}-1$ intraperitoneally). The Schematic diagram of the experimental procedure was shown in Figure 1A. Meanwhile, in order to testify if the renal positive effect of Empa was independent of its glycemia controlling function, we introduced another group of KK-Ay mice which accepted insulin therapy (INS group $n=15$ ) to our study.

2.2. Cellular Experiments 
HK2 cells (purchased from Chinese Academy of Sciences Shanghai Cell Bank, Shanghai, China) were cultured in Dulbecco's Modified Eagle Medium Nutrient Mixture F-12 (DMEM/F-12) supplemented with 10\% fetal bovine serum, $100 \mathrm{U} / \mathrm{ml}$ penicillin, and $100 \mathrm{ug} / \mathrm{ml}$ streptomycin in an atmosphere containing $5 \% \mathrm{CO} 2$ at 37 . HK2 cells were trypsinized and seeded into 6 -well culture plates at a density of $1^{*} 10^{6} /$ well and grown to over $80 \%$ confluence. Serum-free medium containing $0.2 \%$ BSA was cultured overnight to synchronize cells. HK2 cells were treated with normal glucose (NG, $17.5 \mathrm{mmol} / \mathrm{l}$ D-glucose), or high glucose (HG, 33.3 mmol/l D-glucose), or high glucose plus Empa (HG +EMPA, $33.3 \mathrm{mmol} / \mathrm{l} \mathrm{D}$-glucose $+8 \mu \mathrm{g} / \mathrm{ml} \mathrm{Empa)} \mathrm{or}$ high osmotic pressure solution (MA, $17.5 \mathrm{mmol} / \mathrm{l} \mathrm{D}$-glucose $+15.8 \mathrm{mmol} / \mathrm{l} \mathrm{D}$-mannitol).

\subsection{Morphological Analysis of Kidney}

Unilateral renal tissue was fixed with 10\% phosphate-buffered saline (PBS)-buffered formalin and embedded in paraffin. Tissue sections with $5 \mu \mathrm{m}$ thickness were prepared and stained with hematoxylin-eosin (HE) stain. The changes of tissue morphology were observed with an inverted microscope (magnification, 40x; BX51; Olympus Corp., Tokyo, Japan) and captured by the attached camera. All HE stains were performed using five independent histological slides.

\subsection{Western Blotting}

The frozen kidney tissues of mice were lysed with RIPA buffer (Beyotime, Shanghai, China) containing $1 \mathrm{mM}$ phenylmethylsulfonyl fluoride (PMSF, Thermo Fisher Scientific, Inc., cat.No.36978B). Cell lysate of HK2 was treated with RIPA buffer (Beyotime, Shanghai, China) and cocktail (Roche Diagnostics, Mannheim, Germany). The extractions of mitochondrial fractions were obtained by Mitochondria/Cytosol Fractionation Kit (KeyGEN BioTECH, cat.No. KGP8100) according to the manufacturer's protocol. The primary antibodies used in the present study included: AMPK (1:1, 000; Proteintech, cat.No. 10929-2-AP), p-AMPK (1:1, 000; Cell Signaling Technology, \#8208), DRP1 (Cell Signaling Technology, \#8570), DRP1S637 (Abcam, cat. No. ab193216), DRP1S616 (Cell Signaling Technology, \#3455), MFN 2 (Cell Signaling Technology, \#9482), BAX (Proteintech, cat.No. 50599-2-Ig), BCL-2 (Proteintech, cat.No. 12789-1-APS), Cleaved-CASPASE 3 (1:1, 000; Abcam; \#ab2302), Cytochrome C (Proteintech, cat.No.10993-1-AP), Tomm20 homolog (1:1000; Abcam, cat. No. ab56783), SP1 (Proteintech, cat.No.21962-1-AP), PGAM5 (Abcam, cat. No. ab126534), GAPDH (Abcam, cat. No. ab181602). All immunoblot assays were performed using five independent samples.

\subsection{Terminal transferase dUTP nick end labelling assay}

The apoptotic terminal transferase dUTP nick end labelling (TUNEL) assay was performed to detect the DNA fragmentation in the cell nuclei which is a marker of apoptosis. The TUNEL Apoptosis Assay Kit (Beyotime, Shanghai, China) was applied according to the manufacturer's protocol. Each treatment was performed in six replicates.

\subsection{JC-1 Staining}

The mitochondrial potential was tested by a JC-1 probe according to the manufacture's protocol. HK2 Cells were incubated with $10 \mathrm{mg} / \mathrm{ml} \mathrm{JC}-1$ for $10 \mathrm{~min}$ at $37^{\circ} \mathrm{C}$ in the dark and monitored with a fluorescence microscope (magnification, 150x; BX51; Olympus Corp., Tokyo, Japan). The Red-orange fluorescence reflects the potential-dependent dye aggregation in the mitochondria and the green fluorescence represents the monomeric form of JC-1, which suggests the depolarization of the mitochondrial membrane (Reers, Smiley, Mottola-Hartshorn, Chen, Lin \& Chen, 1995). Each treatment was performed in five replicates.

\subsection{Mitotracker Experiments}

The Mitotracker assay (Cell Signaling Technology, \#9082P) was used to label the mitochondria in HK2 cells. HK2 Cells were treated respectively and incubated with Mitotracker (200 nM) for 45 minutes at 37

. Fluorescence signals were detected by confocal fluorescence microscopy (Leica Microsystems, Germany). The data from five independent experiments are presented. 


\subsection{RNA Interference}

Gene silencing was performed by infecting HK2 cells with siRNA oligonucleotides (GenePharma, Shanghai, China). The cells were seeded $\left(5 \times 10^{4}\right.$ cells per well $)$ in six-well plates and incubated for $24 \mathrm{~h}$. The sequences of PGAM5 siRNA (sense 5'-CUG UGC AGU AUU ACG AAG ATT-3', antisense 5'-UCU UCG UAA UAC UGC ACA GTT-3'), SP1 siRNA (sense 5'-CAG CUU GGU AUC AUC ACA ATT-3', antisense 5'-UUG UGA UGA UAC CAA GCU GTT-3') and negative control (sense 5'-UUC UCC GAA CGU GUC ACG UTT-3', antisense 5'-ACG UGA CAC GUU CGG AGA ATT-3') were transfected with lipo2000 Transfection Agent (Invitrogen, USA) according to the manufacturer's protocol. After 48h of the transfection, cells were harvested for next experiments. Each treatment was performed in six replicates.

\subsection{Luciferase Reporter Assay}

A wild-type mouse PGAM5 promoter fragment was amplified by PCR using the primers listed in Figure 5B and cloned into the promoterless pEZX-PG02 (FulenGen Co., Ltd, Guangzhou,China). The online program, JASPAR was used to predict the putative binding sequences of sp1 within the promoter. Deletion mutations in the SP1 binding sequence at -713, -539 and -320 nt were made using the Quikchange II XL kit (Stratagene, La Jolla, CA, USA). To study the involvement of SP1 in the regulation, the wild-type or mutant PGAM5 promoter constructs were cotransfected with the SP1 expression plasmid, or the empty vector into HK2 cells for $36 \mathrm{~h}$ by using X-tremeGENE transfection reagent. The Renilla luciferase reporter plasmid pRL-SV40 (10 ng) was included in the cotransfection mixture. The luciferase assay was performed with a dual-luciferase reporter assay kit (Promega). The result from five independent experiments are presented.

\subsection{Chromatin immunoprecipitation assay}

We conducted the ChIP experiment using ChIP assay Kit (22188S, Cell Signalling Technology, America) according to the manufacturer's protocol. Cell lysates containing soluble chromatin were incubated overnight with anti-SP1 antibody (Proteintech, cat.No.21962-1-AP) , Histone H3 (4620, Cell Signaling Technology, America, provided in the ChIP Assay Kit) and normal mouse IgG (2729, Cell Signaling Technology, America, provided in the ChIP Assay Kit) respectively. Then the immunoprecipitant were purified using spin columns (Cell Signaling Technology) and the de-cross-linked DNA was adopted as templates to PCR amplify mouse PGAM5-specific sequences. The data from five independent experiments are presented.

\subsection{Randomization and blinding}

In the in vivo experiments, the KK-Ay mice were randomly assigned to either Empa group (n=15) or diabetic model group $(n=15)$. All the urinal and serum samples, the created histological slides and tissue specimen were numbered, and the analysis was performed under blinded conditions. In vitro experiments the cells were harvested and numbered, tests were also conducted under blind conditions to reduce the possible operator bias.

\subsection{Statistical Analysis}

The data and statistical analysis comply with the recommendations of the British Journal of Pharmacology on experimental design and analysis in pharmacology (Curtis et al., 2018). Statistical analysis was performed using GraphPad prism7.00 software (GraphPad, RRID:SCR_000306). The normal probability plot was used to examine data distributions. Results are expressed as the mean +- standard deviation. Statistical comparison of two groups was performed by Student's t test and three or more conditions were conducted by one-way ANOVA followed by Tukey's or Sidak's multiple comparison correction, $P<0.05$ was considered as a statistically significant difference.

\section{Results}

\subsection{Renal injury was attenuated in Empa treated mice.}

The Schematic diagram of the experimental procedure was shown in Figure 1A. Remarkable increase of body and kidney weight was detected in KK-AY group and these changes were rescued partially in Empa treated 
mice (Figure 1B, C, E). Meanwhile, the urinary NGAL (ng/24h), and urinary NAG (U/24h), two markers of tubular injury increased obviously in KK-AY group at the $10^{\text {th }}$ week though no significant change of urinary microalbumin $(\mu \mathrm{g} / 24 \mathrm{~h})$ was found (Figure $1 \mathrm{~F}, \mathrm{G}, \mathrm{H})$. With the prolongation of high-fat diet breeding, an obvious increase of $24 \mathrm{~h}$ urinary microalbumin $(\mathrm{ug} / 24 \mathrm{~h})$ was detected in KK-AY group at $16^{\text {th }}$ week, and these trends were reversed in the Empa group (Figure 1 F, G, H). The HE stains of kidney showed obvious tubular vacuolation and dilatation in KK-AY group, and the Empa alleviate these alterations partially (Figure 1D).

\subsection{The apoptosis in KK-Ay mice kidney were alleviated in Empa treated mice.}

Apoptosis is a form of programmed cell death which has been proved to be connected to DKD. In present study, the number of TUNEL positive cells increased dramatically in KK-AY group, and this trend was reversed significantly in Empa group (Fig. 2B). Proteins related to cellular apoptosis were also measured by Western Blotting. We found that in KK-AY group, the expression of pro-apoptotic proteins such as BAX, Cleaved-CASPSE 3 increased significantly, whereas the anti-apoptotic protein BCL-2 reduced obviously, all these changes were reversed in Empa group (Fig. 2A, D). Cytochrome c (CYT), which is released from mitochondria to cytoplasm in the process of apoptosis plays a critical role in the activating of CASPASE. Here we found that the CYT in mitochondria decreased obviously in the KK-AY group and, in the Empa group the mitochondrial CYT recovered (Fig. 2C, D). These above findings suggest that the diabetic environment result in increased apoptosis in kidney and these changes were partially reversed in Empa group.

\subsection{The increased mitochondrial fission in the kidney of KK-Ay mice was rescued in the Empa group.}

To examined the change of mitochondrial dynamics in diabetic environment, we detected the mitochondrial fission related protein such as Drp1, Drp1S616 and DRP1S637 and the pro-fusion protein Mfn2. Here we found no significant difference in Drp1S616 and DRP1 between the KK-AY group and NC group, but the DRP1S637 decreased notably in the KK-AY group (Fig. 2A, E, F). Meanwhile, we found an obvious increase of mito-Drp1 in KK-AY group which verified the transfer of Drp1 from cytosol to mitochondria during the mitochondrial fission. The changes of DRP1S637 and mito-Drp1 in KK-AY group were reversed in Empa treated mice significantly (Fig. $2 \mathrm{C}, \mathrm{E}$ ). There was no remarkable change of MFN2 was found among the three groups (Fig. 2A, G).

3.4. The expression level of AMPK phosphorylation, SP1 and PGAM5 were recovered in Empa treated KK-Ay mice.

We found an obvious decrease of the AMPK phosphorylation and increased PGAM5 expression in KK-AY group and these changes were rescued in the Empa group (Fig. 2F, H). These phenomena remind us a possible connection between the AMPK and PGAM5, thus there may be a mediator correlated the AMPK activation to the PGAM5 expression. By searching the JASPAR online database, we selected the transcription factor $\mathrm{SP} 1$, which is predicted to be bound to the PGAM5 promoter region with high scores for analysis (Fig. $5 \mathrm{~A}$ ). Moreover, we found an opposite trend of change between the AMPK phosphorylation and the SP1 expression, thus the AMPK phosphorylation decreased and the SP1 expression increased in KK-AY group and all the changes were reversed in Empa group (Fig. 2F, H).

Lastly, we examined the changes of apoptosis and mitochondrial fission in the INS group, and we found that though the insulin administration could reverse the hyperglycemia in KK-Ay mice, no obvious alleviation of apoptosis and mitochondrial fission were founded. Meanwhile, no significant changes of SP1, PGAM5 and phosphorylated AMPK were detected. These findings reminded us that controlling hyperglycemia could not alleviate apoptosis and mitochondrial fission in diabetic kidney, thus the Empa's anti-apoptosis and anti-fission effect was independent of its glycemia controlling function.

3.5. The increased apoptosis and mitochondrial fission in HG treated HK2 cell were alleviated in Empa exposed cells.

We next investigated the changes of apoptosis and mitochondrial dynamics in vitro by HK2 cells. According to the CCK8 assay (Supplemental Figure A), we selected the $8 \mathrm{umol} / \mathrm{ml}$ as the testing concentration of 
Empa in our in vitro study. Immunoblotting demonstrated notable increase of pro-apoptotic proteins such as BAX, Cleaved-CASPASE 3 in the HG group. Whereas the anti-apoptotic protein BCL-2 was downregulated (Fig. 3A, E, F). The mito-CYT decreased significantly in the HG group which also proved the increase of apoptosis (Fig. 3C, F). However, all these effects were partially reversed by Empa administration. No significant difference of the BAX, Cleaved-CASPASE 3, mito-Cytc was found between the NG and MA group (Fig. 3A, C, E, F).

We next examined the changes of mitochondrial dynamics among the different groups in vitro. Decreased DRP1S637 expression was noted in HK2 cells subjected to HG, and no change of Drp1 was found (Fig. $3 \mathrm{~A}, \mathrm{G}, \mathrm{H}$ ). Meanwhile, we found an obvious increase of mito-Drp1 in cells subjected to HG (Figs 3C, H) and no notable change of Mfn2 was detected among the four groups (Fig. 3A, G). We also investigated the mitochondrial membrane potential (MMP) with JC-1 staining. We found that the HG treated cells presented with collapsed mitochondrial membrane potential, as evidenced by a higher green fluorescence and lower red fluorescence, then the red fluorescence was recovered in cells subjected to Empa (Fig. 3B). Lastly, we depicted the morphological change of mitochondria with Mito-Tracker Red imaged by laser scanning confocal microscopy, we found that the HG exposed cells showed small punctate and rounded mitochondria. However, mitochondria from Empa-treated cells recovered filamentous and tubular shape again (Fig. 3D).

\subsection{The change of AMPK phosphorylation and the expression of SP1 and PGAM5 were recovered in Empa treated HK2 cells.}

We investigated the change of phosphorylated AMPK, PGAM5 and SP1 protein in vitro. Similarly, we found a remarkable downregulation of phosphorylated AMPK and a significant upregulation of PGAM5 and SP1 expression in HK2 cells subjected to HG, and the Empa recovered these changes significantly (Fig. 4A, F). In order to detect the causality between the AMPK phosphorylation and the SP1-PGAM5 changes, we introduced AMPK activator (AICAR) and inhibitor (Compound C) into our experiments. We found that both HG and Compound C exposure result in decreased phosphorylated AMPK, DRP1S637 and increased SP1 and PGAM5 expression. Both the Empa and the AICAR could alleviate the changes of AMPK, DRP1S637, SP1 and PGAM5 in HK2 cells (Fig. 4C, D, E). Meanwhile, this alleviation effect of Empa diminished again when the Compound $\mathrm{C}$ was administrated (Fig. 4C, D, E).

In order to consolidate the involvement of SP1 and PGAM5 in the AMPK regulated mitochondrial fission, we examined the fission related protein in HG treated HK2 cells that subjected to si-SP1 or si-PGAM5. We found that both si-PGAM5 and si-SP1 significantly reversed the decrease of DRP1S637 in HK2 cells subjected to HG (Fig. 4B, H), but neither si-PGAM5 or si-SP1 could affect the AMPK phosphorylation (Fig. 4B, G). Meanwhile, the si-SP1 obviously reduced the PGAM5 expression but the si-PGAM5 had no effect on SP1 (Fig. 4B, G). The Empa could not only upregulate the phosphorylated AMPK and DRP1S637, but also decrease the expression of SP1 and PGAM5 in HG treated HK2 cells (Fig. 4B, G, H).

\subsection{The SP1 could bind to the promotor region of PGAM5 and increased its transcription in} HK2 cells.

Finally, we further testify our deduction that the SP1 could bind to the PGAM5 promoter region directly. The predicted binding sites within the SP1 sequence are illustrated in Figure 5A. JASPER analysis revealed 3 putative SP1 binding motifs within the promoter of PGAM5 that located at 713, 539, 320 bp upstream of the transcription start (Fig. 5B). We found that overexpression of SP1 significantly increased the protein levels of PGAM5 in HK2 cells (Fig. 5C, D), which confirmed that SP1 can activate the expression of PGAM5. As shown in Fig. 5E, the WT promoter construct of PGAM5 had transcriptional activity in HK2 cells as compared to the promoterless pEZX-PG02. The SP1 remarkably increased the transcriptional activity of the WT promoter. Moreover, the mutant of the SP1 binding site at $-539 \mathrm{nt}$ significantly decreased the transcriptional activity of the PGAM5 promoter. By contrast, the mutant of the SP1 binding site at either -713 or $-320 \mathrm{nt}$ did not alter the promoter activity significantly (Fig. 5E). We next testified the physical interacts between SP1 and PGAM5 promoter by chromatin immunoprecipitation (ChIP) assay using HK2 cells transfected with either SP1 expression construct or its empty vector. DNA from the immunoprecipitates 
was then amplified by PCR with primers located in the promoter region near the putative SP1 binding site. SP1 was found to physically bind to this region, whereas no binding was detected in these samples with the negative control (NC) primers located far from the -539 nt region (Fig. $5 \mathrm{~F}$ ).

\section{Discussion}

Pharmacological intervention targeting SGLT2 has recently attached great concern as a novel therapeutic option for diabetes. Increasing evidences have confirmed the extra renal protective effect of SGLT2 inhibitors beyond the controlling of hyperglycemia.(Perkovic et al., 2019) However, the mechanism through which SGLT2 inhibitors exert the renal protective effect has been rarely reported. Here we find that the Empa can relieve the tubular injury by alleviating the mitochondrial fission though an AMPK-dependent pathway in diabetic mice. By activating AMPK, Empa can remarkably rescues the dephosphorylation of DRP1S637 and inhibits the mitochondria-translocation of DRP1, which eventually result in less mitochondrial fission. The present study revealed the existence of a glycemia independent mechanism of Empa that protects against mitochondrial dysfunction and alleviates the tubular injury in diabetic mice and HK2 cells.

DKD was traditionally considered as a glomerular disease characterized by mesangial expansion, reduction in capillary surface, and podocyte loss which differ it from other glomerulonephritis.(Thomas et al., 2015) However, increasing evidences have revealed the primary contribution of tubular injury in the pathogenesis of DKD.(Bonventre, 2012; Tang \& Lai, 2012) In the present study, we found the urinal biomarkers NAG and NGAL which reflect the early tubular injury increased obviously at the $10^{\text {th }}$ week without change in albuminuria in KK-Ay mice. Since the albuminuria is an early clinical manifestation of DKD and reflects glomerulopathy, our finding verifies the notion that diabetic tubulopathy occurs earlier than glomerular disease.

Recent clinical trials have confirmed the renal protective effect of SGLT2-inhibitors(Mahaffey et al., 2018; Wanner, Inzucchi \& Zinman, 2016). In present study, both the TUNEL and Western Blotting assay consolidated that the Empa could alleviate apoptosis in the kidney of KK-Ay mice. Recent animal and clinical studies have confirmed the critical role of mitochondrial fission in the pathogenesis of DKD.(Galvan et al., 2019; Jiang et al., 2019) However, the signaling pathways by which hyperglycemia leads to mitochondrial fission are not fully understood.(Wang et al., 2012) Here we found that the DRP1S637 decreased significantly and the mitochondria translocation of DRP1 increased remarkably in the kidney of KK-Ay mice. The DRP1 phosphorylation on serine-637 was previously reported to be involved in Parkinson's disease,(Dagda et al., 2011) myocardial ischemia-reperfusion injury(Sharp et al., 2014) and regulation of ER morphology in stressed pancreatic $\beta$-cells through its anti-fission effect.(Wikstrom et al., 2013) Zhou et al. reported that the Empa could rescue the dephosphorylation of DRP1S637 in cardiac microvascular endothelial cells and alleviated the mitochondrial fission, but the intrinsic mechanism remained unclear.(Zhou, Wang, Zhu, Hu, Chen \& Ren, 2018)

To detect the exact mechanism through which the Empa impacts the DRP1 phosphorylation, we conducted a series of investigations. In present study, we found an obviously decreased phosphorylation of AMPK and DRP1S637 in KK-Ay mice kidney, and this change was reversed in the Empa group. We presumed that the Empa may rescue the dephosphorylation of DRP1S637 and alleviate the mitochondrial fission via an AMPK dependent pathway. We also found an obvious increase of SP1 and PGAM5 expression in KK-Ay mice kidney which were reversed in Empa group. These findings remind us that some causative relationship may exist among the AMPK, SP1 and PGAM5.

To verify our deduction, we performed in vitro experiments using HK2 cells. We found that the apoptosis increased significantly in HG treated cells. Meanwhile, the increased mitochondrial fission and disrupted mitochondrial membrane potential were detected in HK2 cells subjected to HG. All these changes were alleviated in Empa treated cells. These findings were consistent with the in vivo experiments.

Then we tested the phosphorylation of AMPK, the expression of SP1 and PGAM5 in HK2 cells to testify our presumption. Here we found that the AMPK activity decreased obviously in HG treated cells and the expressions of SP1 and PGAM5 increased significantly. These changes were rescued in Empa treated 
cells. Those above findings confirmed the involvement of AMPK, SP1 and PGAM5 in the development of mitochondrial fission and the Empa's anti-fission effect. But if there is a causality among these changes was unclear. So, we introduced the AMPK activator and inhibitor to our study. We found that not only the AMPK activator but also the Empa could increase the AMPK phosphorylation. Both the two chemicals could decrease the SP1 and PGAM5 expressions and alleviate the mitochondrial fission. However, the Compound $\mathrm{C}$, an AMPK inhibitor reversed the declining of SP1 and PGAM5 and blocked the anti-fission effect in Empa treated cells. These findings suggested that the AMPK located at the upstream of the AMPK/SP1/PGAM5 axis and played a central role in the process of HG induced mitochondrial fission in HK2 cells.

To further illustrate how the SP1 and PGAM5 participate in the development of the mitochondrial fission, si-SP1 and si-PGAM5 were introduced to our later investigations. We found that both the si-SP1 and si-PGAM5 could alleviated the HG induced mitochondrial fission, but neither of them could activate the AMPK. Meanwhile, the Empa could not only activate the AMPK but also decrease the SP1 and PGAM5 expression and relive the HG induced mitochondrial fission in HK2 cells. These phenomena consolidated our deduction that the AMPK located at the upstream of the AMPK/SP1/PGAM5 axis and the Empa could alleviated the mitochondrial fission via an AMPK dependent pathway. Meanwhile, we found that the si-SP1 could decrease the PGAM5 expression, but the si-PGAM5 had no effect on the SP1 protein. This result suggests that the SP1 could mediate the PGAM5 expression but it is not contrarily. Finally, we verified the direct interaction between the SP1 and the PGAM5 by luciferase reporter assay and chromatin immunoprecipitation (ChIP), and our findings clearly showed that the SP1 could bind to the promotor of PGAM5 at -539 nt and increased its expression.

In conclusion, the present study depicted an AMPK regulated and PGAM5 involved mitochondrial fission pathway in the development of diabetic tubular disease (Figure 6), and we also revealed a glycemia independent mechanism of Empa that protects against the diabetic tubular injury. Our finding could not only provide the experimental and theoretical basis for the expansion of new indications of Empa, but also revealed a new prospective therapeutic target for DKD.

\section{Acknowledgments:}

This work was financially supported by grants from the Natural Science Foundation of Tianjin (18JCYBJC26100 and 18JCZDJC35500), the National Nature Science Foundation of China (81970697) and the National Key Research and Development Program of China (2018YFC1314001).

\section{Competing Interests}

The authors have declared that no competing interest exists.

References:

Bhargava P, \& Schnellmann RG (2017). Mitochondrial energetics in the kidney. Nat Rev Nephrol 13: 629-646.

Bonventre JV (2012). Can we target tubular damage to prevent renal function decline in diabetes? Semin Nephrol 32: 452-462.

Chang CR, \& Blackstone C (2007). Drp1 phosphorylation and mitochondrial regulation. EMBO Rep 8: 1088-1089; author reply 1089-1090.

Curtis MJ, Alexander S, Cirino G, Docherty JR, George CH, Giembycz MA, et al. (2018). Experimental design and analysis and their reporting II: updated and simplified guidance for authors and peer reviewers. Br J Pharmacol 175: 987-993.

Dagda RK, Gusdon AM, Pien I, Strack S, Green S, Li C, et al.(2011). Mitochondrially localized PKA reverses mitochondrial pathology and dysfunction in a cellular model of Parkinson's disease. Cell Death Differ 18: 1914-1923. 
Galvan DL, Long J, Green N, Chang BH, Lin JS, Schumacker P, et al. (2019). Drp1S600 phosphorylation regulates mitochondrial fission and progression of nephropathy in diabetic mice. J Clin Invest 129: 28072823 .

Gregg EW, Li Y, Wang J, Burrows NR, Ali MK, Rolka D, et al.(2014). Changes in diabetes-related complications in the United States, 1990-2010. N Engl J Med 370: 1514-1523.

Gu X, Peng CY, Lin SY, Qin ZY, Liang JL, Chen HJ, et al. (2019). P16(INK4a) played a critical role in exacerbating acute tubular necrosis in acute kidney injury. Am J Transl Res 11: 3850-3861.

Ito T, Tanimoto M, Yamada K, Kaneko S, Matsumoto M, Obayashi K, et al. (2006). Glomerular changes in the KK-Ay/Ta mouse: a possible model for human type 2 diabetic nephropathy. Nephrology (Carlton) $11: 29-35$.

Jiang H, Shao X, Jia S, Qu L, Weng C, Shen X, et al. (2019). The Mitochondria-Targeted Metabolic Tubular Injury in Diabetic Kidney Disease. Cell Physiol Biochem 52: 156-171.

Kang YJ, Bang BR, Han KH, Hong L, Shim EJ, Ma J, et al. (2015). Regulation of NKT cell-mediated immune responses to tumours and liver inflammation by mitochondrial PGAM5-Drp1 signalling. Nat Commun 6: 8371 .

Kilkenny C, Browne W, Cuthill IC, Emerson M, \& Altman DG (2010). Animal research: reporting in vivo experiments: the ARRIVE guidelines. Br J Pharmacol 160: 1577-1579.

Lee JH, Kim JH, Kim JS, Chang JW, Kim SB, Park JS, et al. (2013). AMP-activated protein kinase inhibits TGF-beta-, angiotensin II-, aldosterone-, high glucose-, and albumin-induced epithelial-mesenchymal transition. Am J Physiol Renal Physiol 304: F686-697.

Lu W, Karuppagounder SS, Springer DA, Allen MD, Zheng L, Chao B, et al. (2014). Genetic deficiency of the mitochondrial protein PGAM5 causes a Parkinson's-like movement disorder. Nat Commun 5:4930.

Mahaffey KW, Neal B, Perkovic V, de Zeeuw D, Fulcher G, Erondu N, et al. (2018). Canagliflozin for Primary and Secondary Prevention of Cardiovascular Events: Results From the CANVAS Program (Canagliflozin Cardiovascular Assessment Study). Circulation 137: 323-334.

McGrath JC, \& Lilley E (2015). Implementing guidelines on reporting research using animals (ARRIVE etc.): new requirements for publication in BJP. Br J Pharmacol 172: 3189-3193.

Neal B, Perkovic V, \& Matthews DR (2017). Canagliflozin and Cardiovascular and Renal Events in Type 2 Diabetes. The New England journal of medicine 377: 2099.

Perkovic V, Jardine MJ, Neal B, Bompoint S, Heerspink HJL, Charytan DM, et al. (2019). Canagliflozin and Renal Outcomes in Type 2 Diabetes and Nephropathy. N Engl J Med 380: 2295-2306.

Reers M, Smiley ST, Mottola-Hartshorn C, Chen A, Lin M, \& Chen LB (1995). Mitochondrial membrane potential monitored by JC-1 dye. Methods Enzymol 260: 406-417.

Reidy K, Kang HM, Hostetter T, \& Susztak K (2014). Molecular mechanisms of diabetic kidney disease. J Clin Invest 124: 2333-2340.

Rossello X, \& Yellon DM (2017). The RISK pathway and beyond. Basic research in cardiology 113: 2.

Russo LM, Sandoval RM, Campos SB, Molitoris BA, Comper WD, \& Brown D (2009). Impaired tubular uptake explains albuminuria in early diabetic nephropathy. Journal of the American Society of Nephrology : JASN 20: 489-494.

Sharp WW, Fang YH, Han M, Zhang HJ, Hong Z, Banathy A, et al.(2014). Dynamin-related protein 1 (Drp1)-mediated diastolic dysfunction in myocardial ischemia-reperfusion injury: therapeutic benefits of Drp1 inhibition to reduce mitochondrial fission. Faseb j 28:316-326. 
Steven S, Oelze M, Hanf A, Kroller-Schon S, Kashani F, Roohani S, et al. (2017). The SGLT2 inhibitor empagliflozin improves the primary diabetic complications in ZDF rats. Redox Biol 13: 370-385.

Takiyama Y, Harumi T, Watanabe J, Fujita Y, Honjo J, Shimizu N, et al. (2011). Tubular injury in a rat model of type 2 diabetes is prevented by metformin: a possible role of HIF-1alpha expression and oxygen metabolism. Diabetes 60: 981-992.

Tang SC, \& Lai KN (2012). The pathogenic role of the renal proximal tubular cell in diabetic nephropathy. Nephrol Dial Transplant 27: 3049-3056.

Thomas MC, Brownlee M, Susztak K, Sharma K, Jandeleit-Dahm KA, Zoungas S, et al. (2015). Diabetic kidney disease. Nat Rev Dis Primers 1: 15018.

Toyama EQ, Herzig S, Courchet J, Lewis TL, Jr., Loson OC, Hellberg K, et al. (2016). Metabolism. AMPactivated protein kinase mediates mitochondrial fission in response to energy stress. Science 351: 275-281.

Wang Q, Zhang M, Torres G, Wu S, Ouyang C, Xie Z, et al. (2017). Metformin Suppresses DiabetesAccelerated Atherosclerosis via the Inhibition of Drp1-Mediated Mitochondrial Fission. Diabetes 66:193-205.

Wang W, Wang Y, Long J, Wang J, Haudek SB, Overbeek P, et al.(2012). Mitochondrial fission triggered by hyperglycemia is mediated by ROCK1 activation in podocytes and endothelial cells. Cell Metab 15: 186-200.

Wang Z, Jiang H, Chen S, Du F, \& Wang X (2012). The mitochondrial phosphatase PGAM5 functions at the convergence point of multiple necrotic death pathways. Cell 148: 228-243.

Wanner C, Inzucchi SE, \& Zinman B (2016). Empagliflozin and Progression of Kidney Disease in Type 2 Diabetes. N Engl J Med 375:1801-1802.

Washburn WN, \& Poucher SM (2013). Differentiating sodium-glucose co-transporter-2 inhibitors in development for the treatment of type 2 diabetes mellitus. Expert Opin Investig Drugs 22: 463-486.

Weinberg JM, Venkatachalam MA, Roeser NF, \& Nissim I (2000). Mitochondrial dysfunction during hypoxia/reoxygenation and its correction by anaerobic metabolism of citric acid cycle intermediates. Proceedings of the National Academy of Sciences of the United States of America 97: 2826-2831.

Wikstrom JD, Israeli T, Bachar-Wikstrom E, Swisa A, Ariav Y, Waiss M, et al. (2013). AMPK regulates ER morphology and function in stressed pancreatic beta-cells via phosphorylation of DRP1. Mol Endocrinol 27: $1706-1723$.

Zhou H, Wang S, Zhu P, Hu S, Chen Y, \& Ren J (2018). Empagliflozin rescues diabetic myocardial microvascular injury via AMPK-mediated inhibition of mitochondrial fission. Redox Biol 15: 335-346.

Zinman B, Lachin JM, \& Inzucchi SE (2016). Empagliflozin, Cardiovascular Outcomes, and Mortality in Type 2 Diabetes. The New England journal of medicine 374: 1094.

\section{Figure Legends}

Figure 1. The diabetic tubular injury of KK-Ay mice was alleviated in Empa administrated mice. (A) The Schematic diagram of the experimental procedure.

(B) The appearance of mice. (C) The morphology changes of kidney. (D) Representative micrographs of HE-stained kidney sections. (E) Body weight. (F) urinary NAG, (G) urinary NGAL, and (H) urinary microalbumin. Results are presented as means $\pm \mathrm{SDMs} .{ }^{*} \mathrm{P}<.05$, significantly different as indicated; \# $\mathrm{P}$ $<.05$ significantly different from C57 group;

Figure 2. The apoptosis and mitochondrial fission in KK-Ay mice were alleviated in Empa group, and the AMPK phosphorylation, SP1 and PGAM5 expression in diabetic kidney was reversed in Empa group. (A) The expression of apoptosis, mitochondrial fission and mitochondrial fusion related proteins. (B) The TUNEL assay in kidney. (C) The changes of mitochondrial CYT and DRP1. (D) Quantification of apoptosis 
related protein expressing. (E) Quantitative data of DRP1S637, mito-DRP1 and DRP1S616 expression. (F) The expression of phosphorylated AMPK, SP1 and PGAM5. (G) Quantification of t-DRP1 and MFN2 expression. (H) Quantitative data of phosphorylated AMPK, SP1 and PGAM5. Results are presented as means \pm SDMs. ${ }^{*} \mathrm{P}<.05$, significantly different as indicated; \# $\mathrm{P}<.05$ significantly different from $\mathrm{C} 57$ group;

Figure 3. The Empa alleviated the apoptosis and mitochondrial fission, and recovered the mitochondrial membrane potential in HG treated HK2 cells. (A) The expression of apoptosis, mitochondrial fission and mitochondrial fusion related proteins in HK2 cells. (B) The JC-1 assay in HK2 cells. (C) Changes of mitochondrial CYT and DRP1 in HK2 cells. (D) Change of mitochondria morphology detected by Mito-tracker. (E) Quantitative data of BCL-2/BAX in HK2 cells. (F) Quantification of cleaved-CASPASE 3 and mito-CYT in HK2 cells. (G) Quantitative data of t-DRP1 and MFN2 expression. (H) Quantitative data of DRP1S637 and mito-DRP1 in HK2 cells. Results are presented as means \pm SDMs. ${ }^{*} \mathrm{P}<.05$, significantly different as indicated; \# $\mathrm{P}<.05$ significantly different from NG group;

Figure 4. The Empa alleviated mitochondrial fission through the AMPK-SP1-PGAM5 pathway. (A) The expression of phosphorylated AMPK, SP1 and PGAM5 in HK2 cells. (B) The effect of si-SP1 and siPGAM5 on the DRP1S637 and AMPK phosphorylation. (C) The effect of AMPK activator and inhibitor on the expression of DRP1S637, SP1 and PGAM5. (D) Quantification of SP1 and PGAM5 expression in HG treated HK2 cells that subjected to AMPK activator or inhibitor. (E) Quantification of DRP1S637 and AMPK phosphorylation in HG treated HK2 cells that subjected to AMPK activator or inhibitor. (F) Quantitative data of expression in phosphorylated AMPK, SP1 and PGAM5 in HK2 cells. (G) Quantification of SP1, PGAM5 and phosphorylated AMPK expression in HG treated HK2 cells that subjected to si-SP1 or si-PGAM5. (H) Quantification of DRP1S637 and t-DRP1 expression in HG treated HK2 cells that subjected to si-SP1 or si-PGAM5. Results are presented as means \pm SDMs. ${ }^{*} \mathrm{P}<.05$, significantly different as indicated; \# $\mathrm{P}<.05$ significantly different from NG group (Figure D. E. F) $\# \mathrm{P}<.05$ significantly different from HG group (Figure G. H)

Figure 5. SP1 binds to the promotor of PGAM5 and increased its transcription in HK2 cells. (A) The predicted binding sites to promoters within the SP1 sequence. (B) Putative SP1 binding motifs (bold and underlined) within the promoter of PGAM5. (C, D) Overexpression of SP1 increased the protein level of PGAM5. (E) Mutation of the PGAM5 promoter at 539 nt (Mutant 2) decreased the activity of PGAM5 and deprived its responsiveness to SP1 overexpression. (F) PCR-based ChIP assay confirmed the binding of SP1 to the PGAM5 promoter. Results are presented as means \pm SDMs. \# $\mathrm{P}<.05$ significantly different from Vector;

Figure 6. Proposed model for the signaling pathway by which PGAM5 participates in the diabetic tubular injury via an AMPK dependent pathway.

\section{Supplemental Figure:}

Supplemental Figure 1:The blood glucose and serum insulin changes in different group of mice.A: The blood glucose changes in different group of mice. B: The serum insulin in different group of mice. Results are presented as means \pm SDMs. ${ }^{*} \mathrm{P}<.05$, significantly different as indicated; \# $\mathrm{P}<.05$ significantly different from C57 group;

Supplemental Figure 2: The relative expression of AMPK/SP1/PGAM5 and mitochondrial fission related protein in kidney. Results are presented as means $\pm \mathrm{SDMs} .{ }^{*} \mathrm{P}<.05$, significantly different as indicated; \# $\mathrm{P}<.05$ significantly different from $\mathrm{C} 57$ group;

Supplemental Figure 3: The CCK8 assay which was used to tested the proper concentration of Empa in vitro study. \# $\mathrm{P}<.05$ significantly different from $0 \mathrm{ug} / \mathrm{ml}$. 


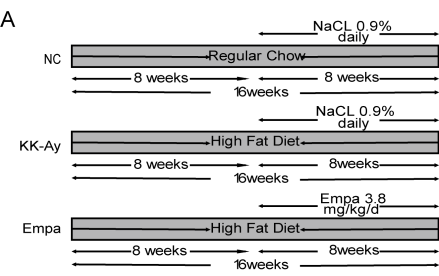

C

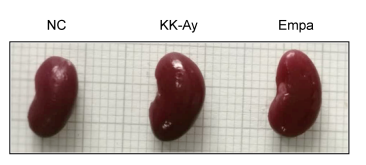

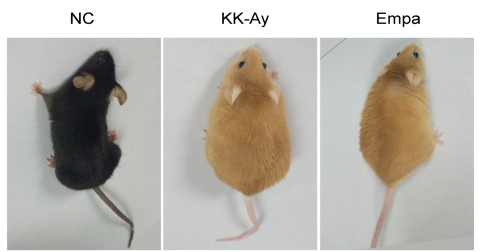

D

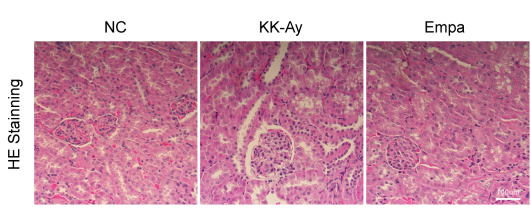

E

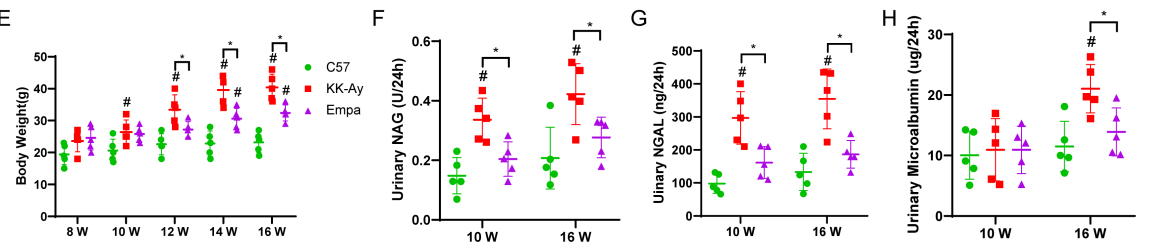

A
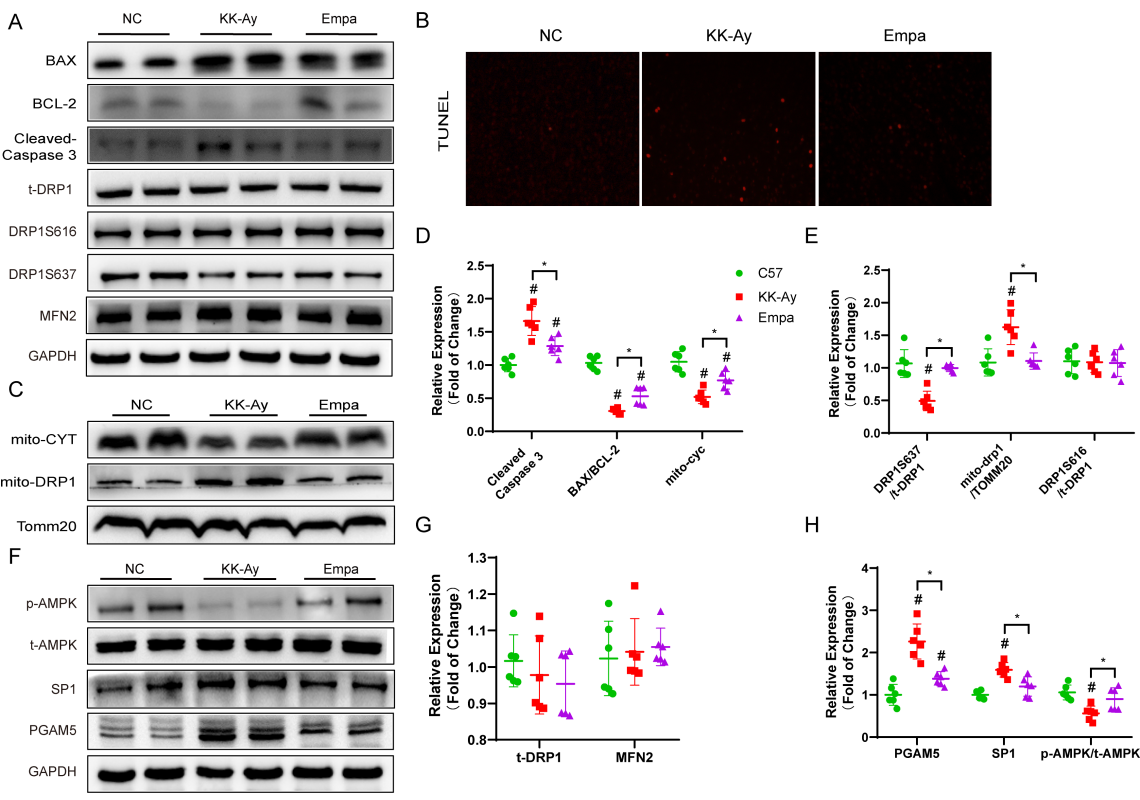
A

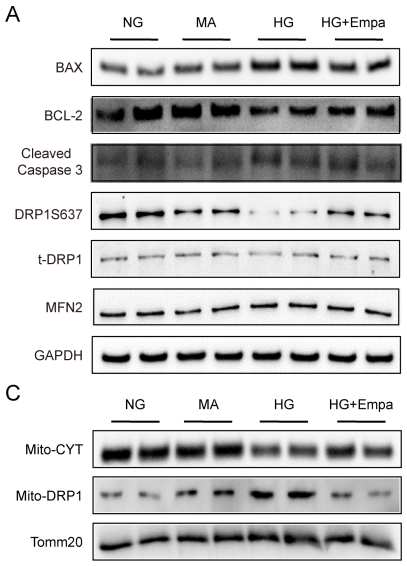

E
B

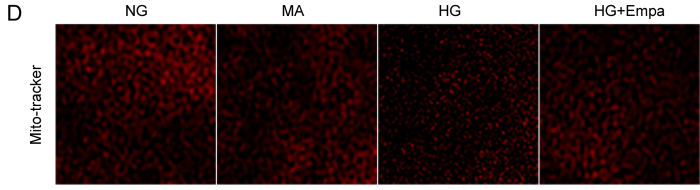

DAPI Mono Jc-1 J-aggregates Merge

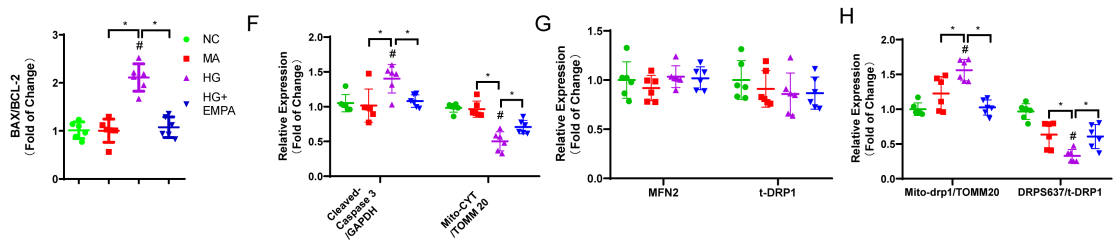

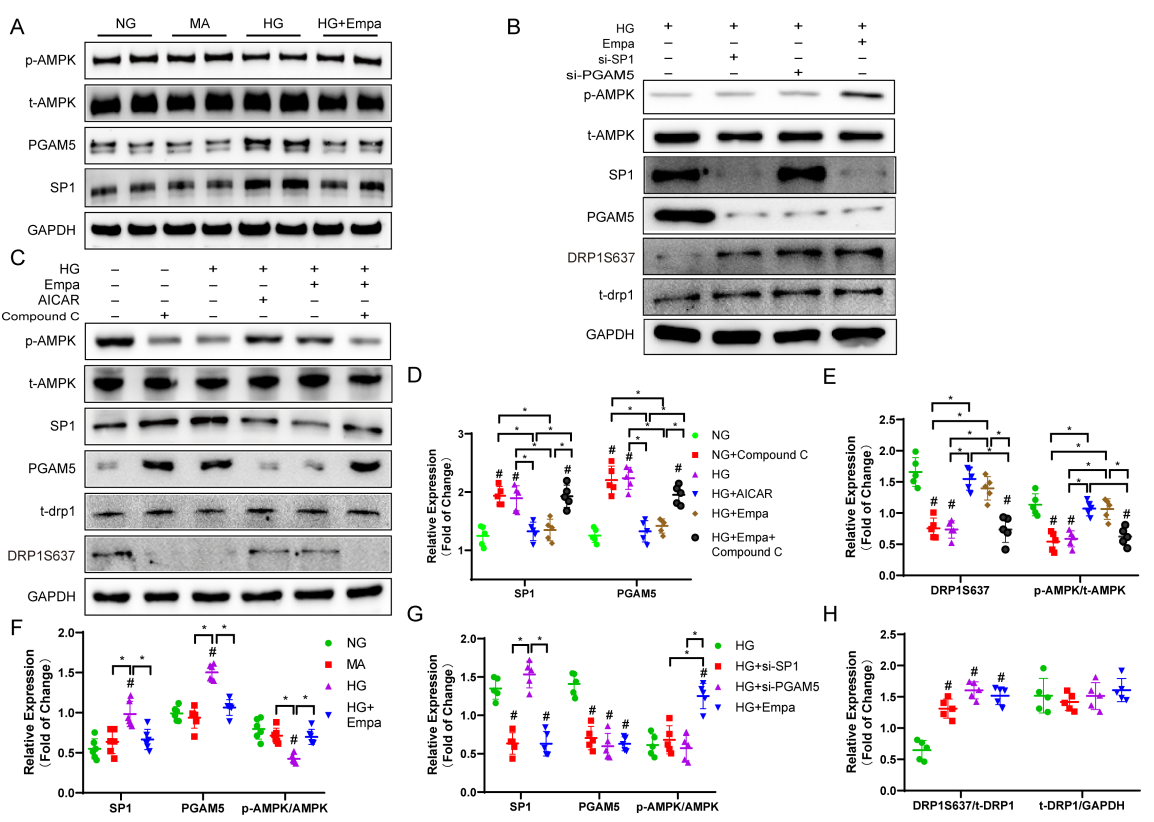


A

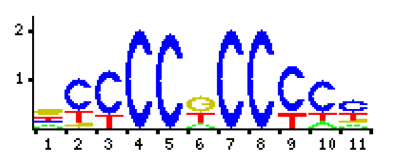

C

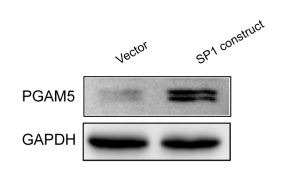

ggcccggggtttgaggggtcgggaccagg gaaggaggggacagccoggccgaactgaacgcacttca ggccgcttgaacagcacacgcaggaactgcgccgacggaaaactttacttcgtgttccgccaccaaa aaatatcgtttaaacaaacctgcggcagcttcccagctcccttaaaccaggatcccaagttctccgcg tctccgcccctgcacccagcctccggcctcggcaggcgcttccggtgcggaaccttctggaaagtacc gcgccgccctcccggcgtccccacaaccccgcccgcacttgcgcgcaccggcgctegcgagcaca gtgacgggactggagcgtggcgcggcaggtggctccacgaaaacgcccettcgcgcaaggctgccce gcgtcctg ccgcgccccaggaggcgggacttccgcccccgccgcccggaagcggcagccggggga agcgtgttcctcgcggagcgcogtcggggccgtgggcgcttgcgcgggccggcgcgggagcaagce gcatggcgttccggcaggcgctgcagctggcggcctgcgggctggccgggggctcggccgccgtgct cttctcggccgtggcggtagga agccgcgcgcaggcggggacgcgag

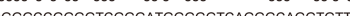

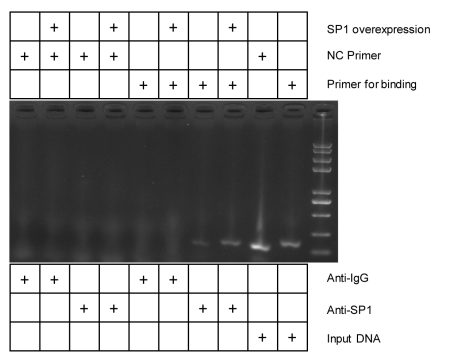

\section{Hyperglycimia}

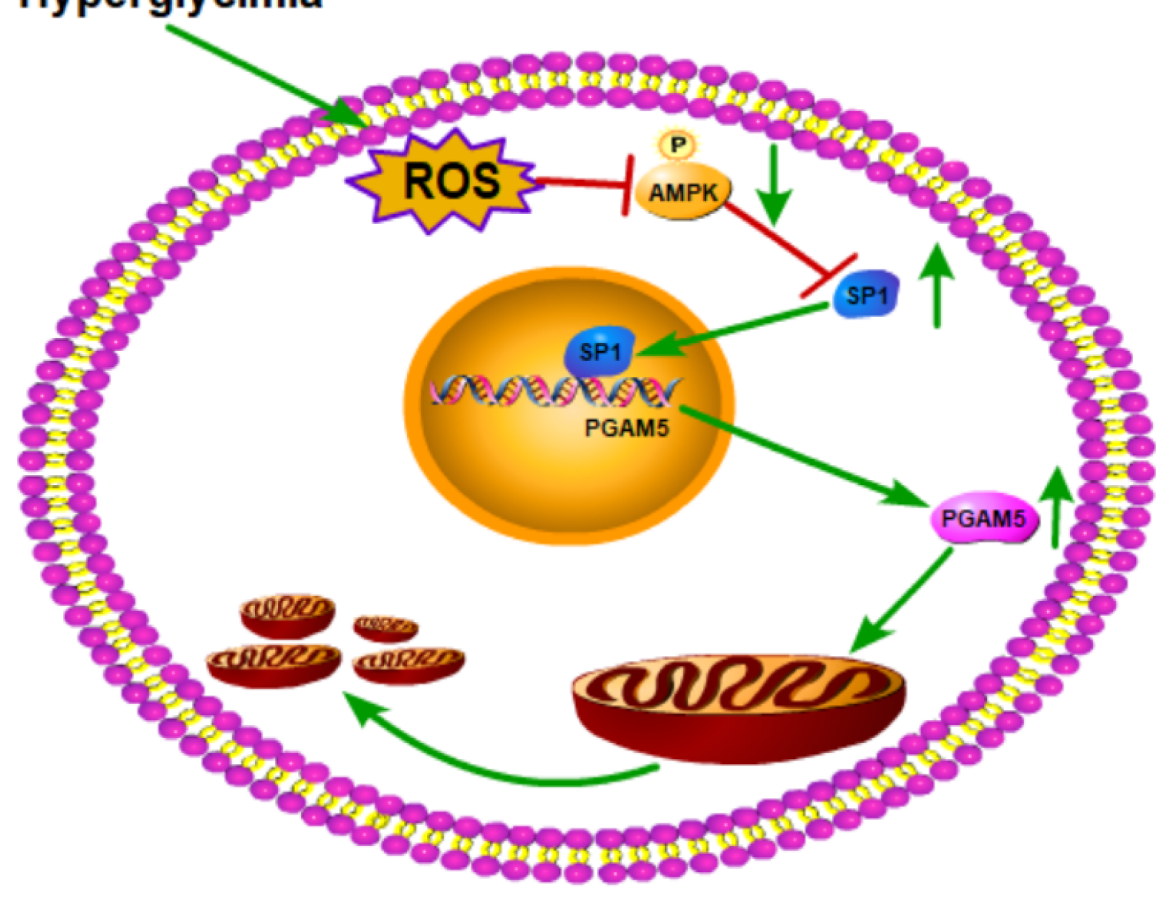

